\title{
Reaction of Sulfur, Hydrogen Sulfide, and Accelerators With Propylene and Butadiene ${ }^{1}$
}

\author{
Frederic J. Linnig, Edwin J. Parks, and Leo A. Wall
}

(September 9, 1960)

\begin{abstract}
As part of a study of vulcanization, propylene as a model compound for natural rubber has been reacted with sulfur alone, with hydrogen sulfide alone, and with each of these materials in the presence of certain accelerators. Butadiene as a model compound for intermediate conjugated systems found in vulcanized rubber by means of infrared studies has been similarly studied. Results of mass spectrometer analyses of the volatile portions of the reaction products indicate the formation of sulfides, disulfides, and carbon-to-carbon bonds. Zinc dimethyl dithiocarbamate (ZnDMDC), a vulcanization accelerator, facilitates the formation of hydrogen sulfide from the olefin or diolefin in the presence of sulfur, and in turn promotes the reaction of hydrogen sulfide with the olefin and diolefin. The $\mathrm{ZnDMDC}$-accelerated reaction of hydrogen sulfide and sulfur with the diolefin may account for the reduced conjugation observed in vulcanizates accelerated with $\mathrm{ZnDMDC}$. Studies with free radical accelerators show that a mechanism other than a free radical chain mechanism is involved in the formation of diisopropyl sulfide in the reaction of propylene with sulfur (or hydrogen sulfide) and certain substances that facilitate the reactions. The same conclusion applies to the formation of a nonvolatile residue in the $\mathrm{ZnDMDC}$-accelerated reaction between propylene and sulfur. Other phases of the reactions involve the formation of compounds from what appear to be free radical fragments of the original molecule. In most of the reactions, appreciable portions of the reaction products are nonvolatile.
\end{abstract}

\section{Introduction}

The processes involved in the vulcanization of natural rubber may be explored from essentially three different standpoints. From changes in the physical properties during or after vulcanization one may deduce that certain changes have occurred in the forces holding the long chain molecules together. Chemical studies, which are necessary to determine the exact nature of these forces or bonds, when applied to the polymer itself, can yield only limited information because of the difficulty of establishing the changes in chemical structure that have taken place. The use of model compounds yields much more detailed data, but it is recognized that these data may not be wholly applicable to the large polymer molecules.

Though much work of all three types has been done in the 121 years since vulcanization was discovered, no general theory of the mechanism of the reaction has as yet been agreed upon. Additional and even contradictory data are continually forthcoming. Since hydrogenated rubber will not vulcanize $[1],^{2}$ it is apparent that the presence of the double bond in rubber must account for its reactivity in the vulcanization process. In the present work propylene was chosen as a model compound for the original olefin present in natural rubber. Its size as one of the smallest compounds that could be chosen for this purpose makes possible convenient analysis

\footnotetext{
1 This work was presented at the 138th meeting of the American Chemical Society, Division of Rubber Chemistry, New York, N.Y., Sept. 1960.
}

Figures in brackets indicate the literature references at the end of this paper. of at least part of the reaction products with the mass spectrometer.

Infrared work in which natural rubber and squalene were reacted with sulfur has indicated the likely occurrence of double bond shifts leading to the presence of 1,4 , and 1,3 diolefins $[2,3]$. Chemical studies with maleic anhydride using infrared analysis verified the presence of the conjugated dienes [2]. Accelerators such as zinc dibutyl dithiocarbamate and tetramethylthiuram disulfide when added to rubber sulfur compounds were found to reduce the amount of conjugated double bonds [2]. More recently ZnDMDC (zinc dimethyl dithiocarbamate) has been found to have a similar effect [3]. Bateman, Glazebrook, and Moore [4] in their work with dihydromyrcene have isolated a conjugated triene, alloocimene. It will be noted that in this case the conjugation is produced not by double bond shifts but by the introduction of a new double bond. In the present work butadiene was chosen as a model compound to determine how the conjugated system, evidently present during vulcanization, would behave as an intermediate. Since hydrogen sulfide has long been considered as an intermediate in the process, its reaction with both olefin and diolefin has also been studied.

The aim of the present work was to identify the volatile products of these reactions and to provide background information for further detailed study of the individual reactions. ZnDMDC has been used as a vulcanization accelerator and di-tert-butyl peroxide and gamma rays have been used as free radical initiators to explore in a general way the mechanisms of the reactions. 


\section{Materials}

Propylene: C. P. Grade, quoted 99.0 percent minimum purity. Mass spectrometer analysis showed about 1.0 to 1.5 percent propane.

Butadiene: Quoted 99.4 percent minimum purity.

Hydrogen sulfide: Purified grade, quoted 99.5 percent minimum purity verified by mass spectrometer analysis.

Sulfur: Purified by the method of Murphy, Clabaugh, and Gilchrist [5].

ZnDMDC (zinc dimethyl dithiocarbamate): Vulcanization accelerator.

Di-tert-butyl peroxide.

\section{Experimental Procedure}

Air and water were removed in the usual manner from the gaseous reagents. Specified amounts of the gaseous materials were introduced into evacuated 3 -mm glass tubes $7 \mathrm{in}$. long into which the required amount of liquid or solid reagent had previously been weighed. Except in the case of the reactant blanks in table 2 , about $6 \times 10^{-4}$ mole of total gas was used per sample in a volume of about $0.44 \mathrm{ml}$. The quantities of reactants given in the tables are approximate.

After reaction at $130^{\circ} \mathrm{C}$ for 4 days, each tube was placed in a glass apparatus equipped with a glass plunger for breaking the $3-\mathrm{mm}$ tubing. The glass apparatus was then evacuated and sealed. The part containing the tube was frozen in liquid nitrogen, the tube broken with the plunger, and the gases allowed to expand into the larger volume to a pressure of about $1 / 4 \mathrm{~atm}$.

The volatile portions of the samples were analyzed in a mass spectrometer. Fractions volatile at liquid nitrogen temperature $\left(-196^{\circ} \mathrm{C}\right)$, dry ice temperature $\left(-78^{\circ} \mathrm{C}\right)$, and room temperature $\left(25^{\circ} \mathrm{C}\right)$ were analyzed separately, pumping off the gases volatile at the lower temperature before distilling off the material volatile at the next higher temperature.

The nonvolatile residue of a sample in which propylene and sulfur were reacted and one in which propylene, sulfur, and $\mathrm{ZnDMDC}$ were reacted, were extracted with $n$-hexane in an attempt to separate the sulfurated reaction product from the remainder of the residue. A few exploratory experiments have been made on the second above-mentioned residue using infrared and gas chromatographic techniques. In other cases a change in the appearance of the original solid or the formation of oily or viscous material has been noted as the formation of a nonvolatile residue.

\section{Results}

Yields of reaction products are presented in tables 1 to 5. Except where more than one isomer of propyl sulfide is indicated, the values in the tables include small proportions of the other isomers of this compound.
It will be noted that the recovery is quite low even in cases where little nonvolatile residue is formed. The reason for this apparent low recovery is not yet clear, but it does not interfere with a qualitative and roughly quantitative interpretation of the data. In spite of the extended reaction period involved, the extent of reaction is sometimes small, a fact probably due to the gas-solid or gas-liquid-solid systems involved.

\subsection{Propylene, Sulfur, and Accelerators}

The results obtained with propylene, sulfur, and accelerators are given in table 1 .

The formation of propadiene in the presence of sulfur involves loss of hydrogen - though no free hydrogen was detected - with the introduction of a new double bond. The fact that propadiene is not formed in the presence of $\mathrm{ZnDMDC}$ is similar to the reduction of conjugation in accelerated rubber vulcanizates [3] assuming, of course, that this conjugation is also produced by the introduction of a new double bond [4]. Diisopropyl sulfide is one of the principal volatile products formed in all four reactions and the quantity formed is not increased in the presence of the free radical accelerator, ditert-butyl peroxide; it is, in fact, notably reduced in amount in the presence of gamma rays, indicating that this product of the reactions may result from something other than a free radical chain mechanism. It might be noted, however, that the work of Moore [6] on the hydrogenation of propylene suggests that isopropyl sulfide radical, if formed, could add to the central carbon atom of propylene and hence produce diisopropyl sulfide. It should be noted that the formation of the 2,5-dimethylthiophene involves the production of a carbon-to-carbon bond.

The reactant "blanks" in table 2 show that carbon disulfide is not found when $\mathrm{ZnDMDC}$ is heated alone or with sulfur, but is found in the presence of propylene. Table 2 indicates that hydrogen sulfide is not detected when ZnDMDC is heated alone but is detected in the presence of sulfur. This last quantity is, however, only about 0.75 percent of the amount obtained in the presence of propylene and sulfur reported in table 1.

Many workers have reported on or discussed the presence or absence of hydrogen sulfide in connection with vulcanization studies using rubber or model compounds under a variety of conditions [7-43]. Wolesensky [44] reviews some of the earlier work in this regard. Wolesensky [44] and Cummings [45] noted the evolution of hydrogen sulfide from vulcanizates between room temperature and the temperature of complete thermal decomposition. Bloomfield [46] and Farmer and Shipley $[34,36]$ discuss the formation of hydrogen sulfide when polysulfides are heated at different temperatures, polysulfides having been reported among the products formed in the reaction of olefins with sulfur $[34,36]$. 
TABLE 1. Reaction of propylene, sulfur, and accelerators ${ }^{\mathrm{a}}$

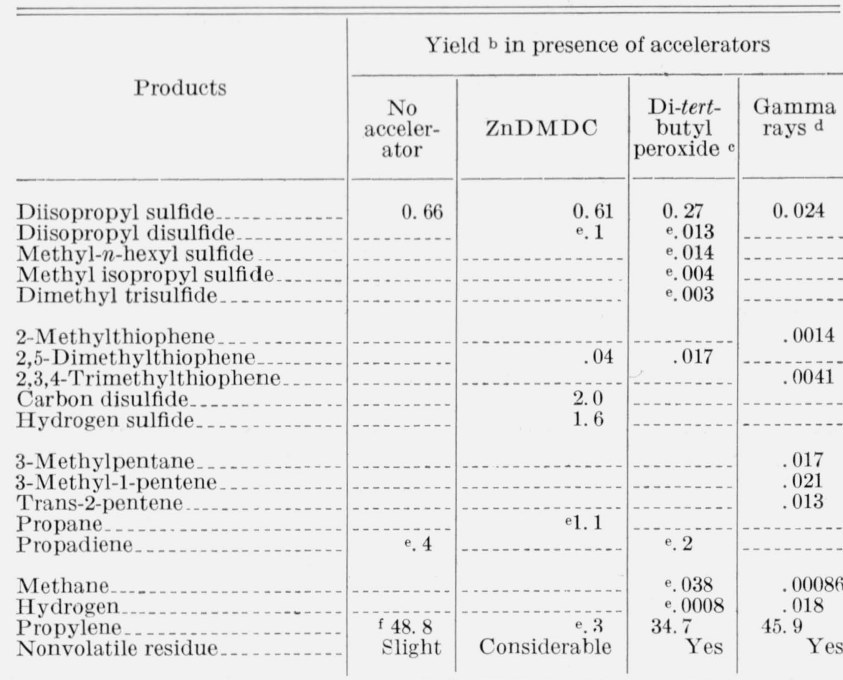

a The materials were heated in sealed tubes at $130{ }^{\circ} \mathrm{C}$ for 4 days. A bout $0.025 \mathrm{~g}$ of propylene was used with about $0.1 \mathrm{~g}$ of sulfur or sulfur plus ZnDMDC (zinc dimethyl dithiocarbamate) in a $2: 1$ ratio. A bout $0.002 \mathrm{~g}$ of di-tert-butyl peroxide was used in the experiment with this catalyst. The reactants were exposed at $130{ }^{\circ} \mathrm{C}$ to a 1700 -curie cobalt-60 source in the gamma radiation experiment

$\mathrm{b}$ Yield number of moles of gaseous product or residual pronylene $\times 100$. initial number of moles of propylene

A verage values of analyses of duplicate tubes are given except where noted c The room temperature fractions of these tubes contained large portions of unidentified material.

d Based on one reaction tube.

- Value based on analysis of one tube; not found in analysis of second tube.

$f$ Value based on analysis of one tube; found in second tube, but not computed quantitatively.

TABLE 2. Reaction of zinc dimethyl dithiocarbamate $(\mathrm{ZnD} M D C)$ with other reactants a

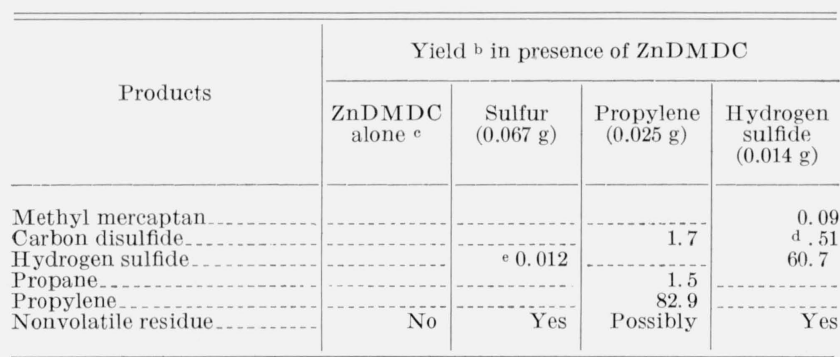

a The materials were heated in sealed tubes at $130{ }^{\circ} \mathrm{C}$ for 4 days. A bout $0.033 \mathrm{~g}$ of $\mathrm{ZnDMDC}$ was used in each experiment.

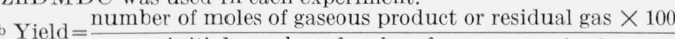

Yield $=\frac{\text { initial number of moles of gaseous reactant }}{\mathrm{C}}$

c Analysis showed small amounts of $\mathrm{C}_{4} \mathrm{H}_{6}$ in all fractions and $\mathrm{C}_{8} \mathrm{H}_{12}$ in the room temperature fraction, evidently as contamination in the spectrometer.

$\mathrm{d}$ The hydrogen sulfide used probably contained about 0.1 percent carbon disulfide as an impurity

e Calculated relative to $0.025 \mathrm{~g}$ of propylene ordinarily used.

Studebaker and Nabors [7] found that purified sulfur produced appreciably less hydrogen sulfide when heated with carbon blacks than did ordinary reagent grade sulfur, and that purified sulfur heated with squalene produced only traces of hydrogen sulfide. The results of Studebaker and Nabors [7] also indicate that some hydrogen sulfide is produced when reagent grade sulfur is heated alone and that this quantity is greater than when purified sulfur is used.
Stevens and Stevens [29] reacted large quantities of sulfur $(200 \text { and } 1000 \mathrm{phr})^{3}$ at $100^{\circ} \mathrm{C}$ for $168 \mathrm{hr}$ in a liquid mixture of $o$ - and $p$-dichlorobenzenes with 20 phr zinc oxide and $20 \mathrm{phr}$ zinc diethyl dithiocarbamate to produce an abundance of hydrogen sulfide, especially in the reaction employing $1000 \mathrm{phr}$ sulfur, presumably due to the large excess of sulfur and the completeness of the reaction with the double bond in rubber. In our work we also used an excess of sulfur as well as long reaction periods, and in the presence of $\mathrm{ZnDMDC}$ recovered very little propylene.

The quantity of propane reported in column 2 of the numerical data in table 1 is close to the amount present as an impurity in the original propylene as indicated by mass spectrometric analysis.

As indicated above, ZnDMDC markedly reduces the quantity of residual propylene, thus increasing the amount of nonvolatile residue, whereas a heavy dose of gamma rays does not affect greatly the extent of overall reaction. However, there appears to be some reduction in the amount of residual propylene in the presence of the perioxide used in small amounts. Nevertheless, in the overall view, there is little evidence of a free radical chain mechanism being involved in the formation of the nonvolatile residue in the tube containing $\mathrm{ZnDMDC}$. Infrared spectra of this nonvolatile residue indicate that it is not merely polypropylene. Gas chromatographic separation of the portion of this residue extractable with $n$-hexane yields at least three very broad bands and the pyrolyzate of this same material yields about 12 peaks, both results indicating a material of high molecular weight and perhaps a high degree of complexity.

The presence of methyl- $n$-hexyl sulfide, methyl isopropyl sulfide, and dimethyl trisulfide in the reaction in which the peroxide was used, suggests that free radicals do aid in the formation of sulfur compounds through fragmentation and rearrangement. However, some of the fragments may have come from the peroxide itself. The formation of methane and hydrogen in the presence of both free radical accelerators and the formation of 3-methylpentane, 3-methyl-1-pentene, and trans-2-pentene in the presence of gamma rays are evidences of reactions not involving sulfur.

\subsection{Propylene, Hydrogen Sulfide, and Accelerators}

The results obtained by reacting propylene with hydrogen sulfide and accelerators are given in table 3 .

Little reaction takes place in the absence of an accelerator in agreement with previous findings [47] even when reaction was attempted at $180{ }^{\circ} \mathrm{C}$. Naylor [33] gives references in which a variety of "catalysts" were employed for this type of reaction. The presence of a small amount of sulfur produces more diisopropyl sulfide than was produced in the reaction between propylene and sulfur alone (though the sulfur was present in larger amounts than here),

\footnotetext{
${ }^{3} \mathrm{phr}=$ parts per hundred parts of rubber.
} 
indicating the effect of this element in promoting the reaction between propylene and hydrogen sulfide. Some isopropyl mercaptan may also be formed (see footnote $\mathrm{c}$ of table 3 ). These results are in agreement with those of Jones and Reid [47] obtained at $180{ }^{\circ} \mathrm{C}$ except that they do not report finding methyl ethyl sulfide, ethane, and ethylene. $\mathrm{ZnDMDC}$ produces an appreciable quantity of diisopropyl sulfide.

TABLE 3. Reaction of propylene, hydrogen sulfide, and accelerators a

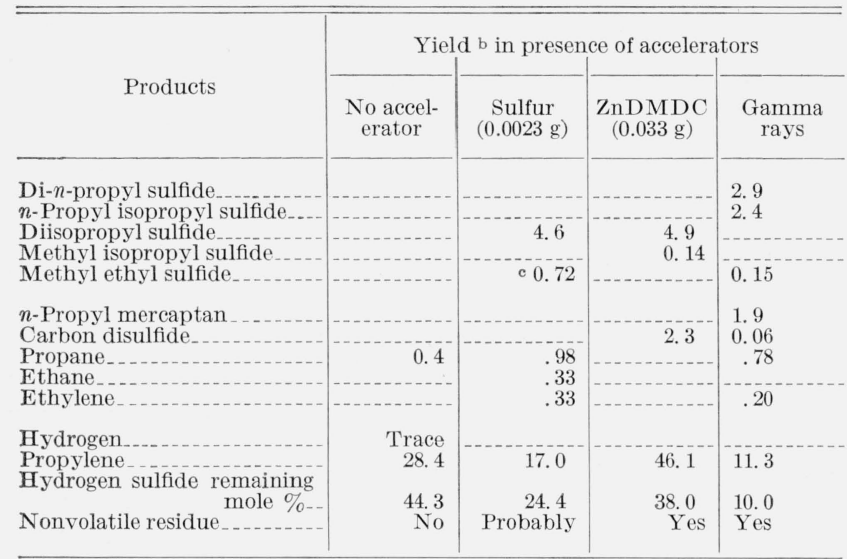

a The materials were heated in sealed tubes at $130^{\circ} \mathrm{C}$ for 4 days. About $0.017 \mathrm{~g}$ of propylene was used with about $0.007 \mathrm{~g}$ of hydrogen sulfide in each experiment. The reactants were exposed at $130^{\circ} \mathrm{C}$ to a 1700 -curie cobalt-60 source in the gamma radiation experiment.

\footnotetext{
b Yield $=\underline{\text { number of moles of gaseous product or residual propylene } \times 100}$ initial number of moles of propylene

c Analysis shows about 50 percent of this constituent could be isopropyl mer-
} captan.

The information presented in section 4.1 indicates that one function of $\mathrm{ZnDMDC}$ in vulcanization may be to produce hydrogen sulfide in the presence of sulfur and the olefin. The data in table 3, if applied to rubber, suggest that $\mathrm{ZnDMDC}$ will also promote the reaction of the double bond with any hydrogen sulfide formed during ordinary vulcanization. This last reaction coupled with the "catalytic" effect of sulfur should keep the amount of free hydrogen sulfide at a low level because it is formed and reacted in the rubber where it cannot readily escape.

Failure to find additional diisopropyl sulfide when $\mathrm{ZnDMDC}$ was used in the propylene sulfur reaction appears to be due to the fact that too little hydrogen sulfide was formed to affect appreciably the reaction in this type of system. Furthermore some of the reaction product of $\mathrm{ZnDMDC}$, propylene, and hydrogen sulfide in the presence of sulfur may be nonvolatile and appear in the residue, as it also seems to do in column 3 of the numerical data in table 3. Many workers have suggested a variety of ways in which hydrogen sulfide could play a part in vulcanization $[7-9,11,13-15,18-21,23-28,30-41$, 48-51].

The amount of carbon disulfide in column 3 of the numerical data is about equal to that formed in the propylene-ZnDMDC, and hydrogen sulfideZnDMDC "blanks" combined (table 2).

The formation of $n$-propyl mercaptan, di- $n$-propyl sulfide, and $n$-propyl isopropyl sulfide in the presence of gamma rays indicates a considerable amount of abnormal addition contrary to Markownikoff's rule, including the formation of $n$-propyl mercaptan as an intermediate in the production of the sulfides. This type of reaction has for some time been considered as evidence of a free radical chain mechanism $[30,52,53]$. The formation of diisopropyl sulfide with either sulfur or $\mathrm{ZnDMDC}$ is an indication of a mechanism other than a free radical chain reaction. A polar mechanism was indicated by Naylor [30] for the "sulfur-catalyzed" reaction of hydrogen sulfide with an olefin.

Breakdown of the original propylene is indicated by the formation of hydrogen (if not present as a residual gas in the spectrometer) in the first column of numerical data, the formation of methyl ethyl sulfide, ethane, and ethylene in the second column, and the formation of methyl isopropyl sulfide in the third column. The methyl fragment producing the methyl isopropyl sulfide in the third column may have come from the $\mathrm{ZnDMDC}$. The formation of methyl ethyl sulfide, carbon disulfide, and ethylene is evidence of breakdown in the presence of gamma rays.

Some nonvolatile residue is formed in the presence of $\mathrm{ZnDMDC}$, but the low recovery of hydrogen sulfide and propylene in the presence of gamma rays suggests that a large portion of these reactants has produced a nonvolatile product.

\subsection{Butadiene, Sulfur, and ZnDMDC}

The results obtained when butadiene is reacted with sulfur alone and with sulfur and $\mathrm{ZnDMDC}$ are given in table 4.

TABLE 4. Reaction of butadiene, sulfur, and zinc dimethyl dithiocarbamate $(Z n D M D C)^{\text {a }}$

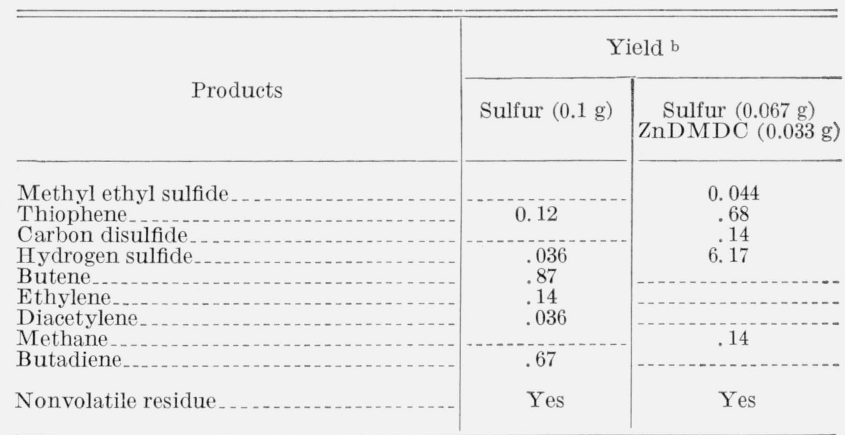

a The materials were heated in sealed tubes at $130^{\circ} \mathrm{C}$ for 4 days. About $0.032 \mathrm{~g}$ of butadiene was used in each experiment.

b Yield $=$ number of moles of gaseous product or residual butadiene $\times 100$. initial number of moles of butadiene

The formation of thiophene is facilitated somewhat by $\mathrm{ZnDMDC}$. The relatively large quantity of hydrogen sulfide formed in the presence of $\mathrm{ZnDMDC}$ is consistent with the results of the propylene studies 
In this case the carbon disulfide is probably also related to a reaction between an olefin and $\mathrm{ZnDMDC}$ as indicated by the propylene-ZnDMDC "blank" in table 2. The remaining compounds formed in both reactions appear to result from fragmentation of the original butadiene, rearrangement, and hydrogen migration. Again, the methyl group in methyl ethyl sulfide may result from decomposition of the $\mathrm{ZnDMDC}$.

Some residual butadiene exists in the presence of sulfur alone, but none when $\mathrm{ZnDMDC}$ is also present. This result is consistent with the reduced conjugation present in vulcanizates accelerated with $\mathrm{ZnDMDC}$ [3]. However, the opportunity for polymerization of the conjugated system in vulcanizing rubber should be much less than in the case of butadiene itself and should lead to larger equilibrium concentrations of conjugated systems in both types of vulcanizates. In the present work, the polymerized butadiene along with nonvolatile sulfur compounds is assumed to be present in the residues of the samples listed in table 4. Shepard, Henne, and Midgley [54] have prepared thiophene and its homologs in 6-50 percent yield by reacting 1,3 diolefins with sulfur at about $350{ }^{\circ} \mathrm{C}$. Byproducts of the reaction at this temperature were hydrogen sulfide, carbon disulfide, and nonvolatile materials. Böttcher and Lüttringhaus [55] have prepared a trithione $\mathrm{C}_{5} \mathrm{H}_{6} \mathrm{~S}_{3}$ in 5 percent yield from isoprene and sulfur.

\subsection{Butadiene, Hydrogen Sulfide, and Accelerators}

The results obtained when butadiene is reacted with hydrogen sulfide alone and in the presence of $\mathrm{ZnDMDC}$ and di-tert-butyl peroxide are given in table 5.

\section{TABLE 5. Reaction of butadiene, hydrogen sulfide, and} accelerators a

\begin{tabular}{|c|c|c|c|}
\hline \multirow{2}{*}{ Products } & \multicolumn{3}{|c|}{ Yield $\mathrm{b}$ in presence of accelerators } \\
\hline & $\begin{array}{l}\text { No acceler- } \\
\quad \text { ator }\end{array}$ & $\underset{(0.033 \mathrm{~g})}{\mathrm{ZnDMDC}}$ & $\begin{array}{l}\text { Di-tert-buty } \\
\text { peroxide } \\
(0.002 \mathrm{~g})\end{array}$ \\
\hline $\begin{array}{l}\text { Di-tert-butyl sulfide } \\
\text { Methyl ethyl sulfide } \\
\text { Ethyl mercaptan } \\
\text { Carbon disulfide } \\
\text { tert-Butanol }\end{array}$ & $\begin{array}{r}0.05 \\
.18\end{array}$ & 0.66 & \begin{tabular}{c}
0.06 \\
\hdashline 2.9
\end{tabular} \\
\hline $\begin{array}{l}\mathrm{C}_{8} \mathrm{H}_{14} \\
\mathrm{C}_{8} \mathrm{H}_{12} \\
n \text {-Butane } \\
\mathrm{C}_{4} \mathrm{H}_{8} \\
\text { Methane }\end{array}$ & 3.3 & 1.03 & $\begin{array}{l}0.02 \\
.064 \\
.55 \\
.25\end{array}$ \\
\hline $\begin{array}{l}\text { Butadiene } \\
\text { Hydrogen sulfide remaining } \ldots \text { mole } \%\end{array}$ & $\begin{array}{r}3.9 \\
54.3\end{array}$ & d 0.084 & $\begin{array}{r}\text { d } .21 \\
.62\end{array}$ \\
\hline Nonvolatile residue.. & Probably & Yes & Yes \\
\hline
\end{tabular}

a The materials were heated in sealed tubes at $130^{\circ} \mathrm{C}$ for 4 days. About $0.022 \mathrm{~g}$ of butadiene, and $0.007 \mathrm{~g}$ hydrogen sulfide was used in each experiment.

b Yield $=\frac{\text { number of moles of gaseous product or residual butadiene } \times 100}{\text { initial number of moles of butadiene }}$.

e The room temperature fraction of this tube contained a large portion of unidentified material

d Assumed on logical grounds to be 1,3-butadiene; mass spectrometer analysis indicates possibility of its being 1,2-butadiene.
The formation of methyl ethyl sulfide and ethyl mercaptan when the two gases are reacted alone gives evidence of some reaction in contrast with the results obtained with propylene. Their formation also shows evidence of fragmentation and hydrogen migration. The small amount of residual butadiene and the presence of the unsaturated hydrocarbon $\mathrm{C}_{8} \mathrm{H}_{12}$ give evidence of the tendency of butadiene to polymerize and dimerize under these conditions. The relatively large quantity of residual hydrogen sulfide indicates that the major portion of the nonvolatile residue is polymer and not sulfurated reaction product.

The presence of ZnDMDC produces as usual carbon disulfide as well as a butene. Nearly all of the butadiene and all of the hydrogen sulfide seem to have produced a nonvolatile residue. It is apparent that this nonvolatile residue is not entirely polymeric in nature and must contain nonvolatile sulfurated products. Apparently ZnDMDC also promotes the reaction of hydrogen sulfide with butadiene.

The presence of the peroxide leads to the formation of di-tert-butyl sulfide and tert-butyl alcohol probably as a result of decomposition of the di-tert-butyl peroxide. The unsaturated hydrocarbon $\mathrm{C}_{8} \mathrm{H}_{14}$ is also formed as well as mixed butenes, normal butane, and methane, presumably involving fragmentation and hydrogen migration. Nearly all the butadiene and hydrogen sulfide appear to have produced a nonvolatile residue that must necessarily contain a considerable proportion of sulfurated material. Ditert-butyl peroxide, like $\mathrm{ZnDMDC}$ strongly promotes the reaction of the diolefin and hydrogen sulfide, suggesting that $\mathrm{ZnDMDC}$ acts to produce a free radical chain mechanism.

Another sample not reported in table 5 contained both sulfur and ZnDMDC as accelerators of the reaction between butadiene and hydrogen sulfide. In computing the spectrum it was possible to take out about a dozen sulfurated products in addition to the usual carbon disulfide. These included a variety of sulfides (mono, di, and tri), a mercaptan from fragments of the original butadiene, thiophenes, and substituted thiophenes. The products seem to have resulted from ring closure, fragmentation of the original butadiene, rearrangement, and hydrogen migration. In this case, considerable hydrogen sulfide seemed to have been formed. Schneider, Bock, and Häusser [56] have also obtained thiophenes by reacting butadiene with hydrogen sulfide at temperatures between 420 and $600{ }^{\circ} \mathrm{C}$ using pyrites as a catalyst.

\section{Discussion}

Extensive detailed study of the individual reactions, especially of the nonvolatile portions of the reaction products, will of course be necessary before any definite overall mechanism may be deduced, but the present study suggests certain conclusions of a general nature. The application of these conclusions to typical vulcanization reactions may be limited not only by the small size of the model compounds 
but also by the fact that, in order to allow ample opportunity for reaction to occur in the systems studied here, large proportions of sulfur and $\mathrm{ZnDMDC}$ were used, and the reactions were carried out for four days at vulcanization temperatures. Furthermore, it is not known to what extent conjugated double bonds are produced before they react as intermediates.

The compounds found, including those produced through fragmentation and rearrangement indicate the formation of at least mono and disulfides and carbon-to-carbon bonds which may be sources of cross links in vulcanized rubber. Small amounts of thiophenes appear to result from the conjugated systems acting as intermediates as well as a certain amount of polymerization. Hydrogen sulfide is probably formed during vulcanization, and its formation in the reaction between sulfur and the olefin or conjugated system is probably promoted by the vulcanization accelerator $\mathrm{ZnDMDC}$. Both $\mathrm{ZnDMDC}$ and sulfur promote the reaction of hydrogen sulfide with the olefin. The effect of $\mathrm{ZnDMDC}$ on the reaction of the conjugated system with sulfur and with hydrogen sulfide may account for the reduced amount of conjugation in vulcanizates accelerated with $\mathrm{ZnDMDC}$ [3].

The observed effect of free radical accelerators on the formation of diisopropyl sulfide in the reaction of propylene and sulfur and propylene, sulfur, and $\mathrm{ZnDMDC}$ as well as the normal mode of addition found in the reaction of propylene with hydrogen sulfide in the presence of sulfur or $\mathrm{ZnDMDC}$ indicate that something other than a free radical chain mechanism is involved in these reactions. There is very little evidence that free radicals initiate the formation of the nonvolatile residue in the reaction of propylene with sulfur and $\mathrm{ZnDMDC}$, suggesting that this phase of the reaction also involves something other than a free radical chain mechanism. However, a free radical mechanism not involving a chain reaction cannot be ruled out in these cases.

In phases of the reactions in which compounds have been formed through fragmentation and rearrangement, the presence of free radicals as intermediates is at least suggested. The reaction of butadiene with hydrogen sulfide in the presence of $\mathrm{ZnDMDC}$ to form a nonvolatile residue may be a chain reaction involving free radicals. Linnig and Florin [57] have observed electron spin resonance absorption in rubber-sulfur vulcanizates and in rubber heated alone in air under vulcanizing conditions, indicating the presence of free radicals.

The authors are indebted to Edith I. Quinn and Florence R. McCann, formerly of the Bureau, and to Sharon G. Lias for their invaluable efforts in making the mass spectrometer analyses.

\section{References}

[1] H. Staudinger and J. Fritsche, Helv. Chim. Acta 5, 785 (1922).

[2] F. J. Linnig and J. E. Stewart, J. Research NBS 60, 9 (1958) RP2816.

[3] J. E. Stewart and F. J. Linnig, unpublished work, Beckman Instruments, Inc., and NBS.

[4] L. Bateman, R. W. Glazebrook, and C. G. Moore, J. Chem. Soc. 579, 2846 (1958).

[5] T. J. Murphy, W. S. Clabaugh, and Raleigh Gilchrist, J. Research NBS 64A, 355 (1960).

[6] W. J. Moore, J. Chem. Phys. 16, 916 (1948).

[7] M. L. Studebaker and L. G. Nabors, Rubber Age 80, 837 (1957).

[8] G. J. van Veersen, Rev. gén. caoutchouc 28, 411, 636 (1951).

[9] David Craig, A. E. Juve, W. L. Davidson, W. L. Semon, and D. C. Hay, J. Polymer Sci. 8, 321 (1952); Rubber Chem. and Technol. 26, 57 (1953).

[10] David Craig, Dwain Diller, and E. H. Rowe, J. Polymer Sci. 28, 435 (1958).

[11] David Craig, Rubber Chem. and Technol. 30, 1291 (1957).

[12] H. L. Fisher and Y. Schubert, Ind. Eng. Chem. Ind. Ed. 28, 209 (1936); Rubber Chem. and Technol. 9, 350 (1936).

[13] H. L. Fisher, Ind. Eng. Chem. Ind. Ed. 31, 1381 (1939); Rubber Chem. and Technol. 13, 50 (1940).

[14] A. van Rossem, India Rubber J. 91, 845 (1936); Rubber Chem. and Technol. 10, 203 (1937).

[15] T. R. Griffith and D. W. MacGregor, Ind. Eng. Chem. 45, 380 (1953); Rubber Chem. and Technol. 26, 716 (1953).

[16] Tadao Okita, J. Soc. Rubber Ind., Japan 12, 586 (1939).

[17] Tadao Okita, J. Soc. Rubber Ind., Japan 14, 363 (1940).

[18] Tadao Okita, J. Soc. Rubber Ind., Japan 13, 399 (1940); English abstract, ibid. 13, 731 (1940).

[19] Tadao Okita and Yoshiro Shiiba, J. Soc. Rubber Ind., Japan 21, 102 (1948).

[20] Tadao Okita, J. Soc. Chem. Ind., Japan 41, Supplemental binding 207B (1938); Rubber Chem, and Technol. 12, 71 (1939)

[21] Tadao Okita, J. Soc. Chem. Ind., Japan 41, Supplemental binding 205B (1938); Rubber Chem. and Technol. 12, 78 (1939).

22] Seiiti Numajiri, J Soc. Chem. Ind, Japan 45, 844 (1942)

[23] S. G. Zhavoronok, J. Appl. Chem. USSR (Zhur. Priklad. Khim.) 9, 1290 (1936).

[24] E. A. Hauser and J. R. Brown, Ind. Eng. Chem. Ind. Ed. 31, 1225 (1939); R ubber Chem. and Technol. 13, $65(1940)$

[25] R. T. Armstrong, J. R. Little, and K. W. Doak, Ind. Eng. Chem. Ind. Ed. 36, 628 (1944); Rubber Chem. and Technol. 1\%, 788 (1944).

[26] C. W. Bedford and Harold Gray, Ind. Eng. Chem. Ind. Ed. 15, 720 (1923).

[27] C. W. Bedford and H. A. Winkelmann, Ind. Eng. Chem. Ind. Ed. 16, 32 (1924).

[28] C. W. Bedford and L. B. Sebrell, Ind. Eng. Chem. Ind. Ed. 14, 25 (1922)

[29] H. P. Stevens and W. H. Stevens, J. Soc. Chem. Ind. (London) 48, 55T (1929); Rubber Chem. and Technol. 2, 421 (1929)

[30] R. F. Naylor, J. Polymer Sci. 1, 305 (1946); Rubber Chem. and Technol. 20, 353 (1947).

[31] G. F. Bloomfield, India Rubber J. 111, 277, 313 (1946).

[32] G. F. Bloomfield J. Soc. Chem. Ind. (London) 67, 14 (1948); Rubber Chem. and Technol. 21, 543 (1948).

[33] R. F. Naylor, J. Chem. Soc. 1532 (1947).

[34] E. H. Farmer and F. W. Shipley, J. Chem. Soc. 1519 (1947).

[35] G. F. Bloomfield, J. Polymer Sci. 1, 312 (1946); Rubber Chem. and Technol. 20, 360 (1947). 
[36] E. H. Farmer and F. W. Shipley, J. Polymer Sci. 1, 293 (1946); Rubber Chem. and Technol. 20, 341 (1947).

[37] G. J. van Amerongen and R. Houwink, J. Prakt. Chem. 161, 261 (1943); Rubber Chem. and Technol. 16, 834 (1943).

[38] E. B. Newton, Ind. Eng. Chem. Ind. Ed. 34, 374 (1942); Rubber Chem. and Technol. 15, 580 (1942).

[39] G. F. Bloomfield and R. F. Naylor, Proceedings of the XIth International Congress of Pure and Applied Chemistry Vol. II, p. 7, Organic Chem., Biochem. (London, England, 1947)

[40] R. F. Naylor, India Rubber J. 114, 513, 521 (1948).

[41] E. H. Farmer, J. F. Ford, and J. A. Lyons, J. Appl. Chem 4, 554 (1954); Rubber Chem. and Technol. 28, 470 (1955).

[42] C. M. Hull, S. R. Olsen, and W. G. France, Ind. Eng. Chem. Ind. Ed. 38, 1282 (1946); Rubber Chem. and Technol. 20, 627 (1947).

43] NBS, J. Franklin Inst. 219, 607 (1935).

[44] Edward Wolesensky, BS J. Research 4, 501 (1930) RP 162 Rubber Chem. and Technol. 3, 386 (1930).

[45] A. D. Cummings, BS J. Research 9, 163 (1932) RP 464; Rubber Chem, and Technol. 6, 46 (1933).

[46] G. F. Bloomfield, J. Chem. Soc., 1547 (1947); Rubber Chem. and Technol. 22, 348 (1949).
[47] S. O. Jones and E. E. Reid, J. Am. Chem. Soc. 60, 2452 (1938).

[48] E. W. Booth and D. J. Beaver, Ind. Eng. Chem. Ind. Ed. 32, 1006 (1940) ; Rubber Chem. and Technol. 13, 918 (1940).

[49] E. C. B. Bott, Trans. Inst. Rubber Ind. 16, 35 (1940); Rubber Chem. and Technol. 14, 45 (1941).

[50] Yutaka Kawaoka, J. Soc. Rubber Ind. Japan 16, 673 (1943).

[51] B. V. Byzov and M. K. Popova, J. General Chem. (USSR) 4, 889 (1934); Rubber Chem. and Technol. 8, 437 (1935).

[52] W. E. Vaughan and F. F. Rust, J. Org. Chem. 7, 472 (1942).

[53] F. R. Mayo and Cheves Walling, Chem. Revs. 27, 387 (1940).

[54] A. F. Shepard, A. L. Henne, and Thomas Midgley Jr., J. Am. Chem. Soc. 56, 1355 (1934).

[55] Bruno Böttcher and Arthur Lüttringhaus, Ann. 557, 89 (1947).

[56] G. G. Schneider, H. Bock, and H. Häusser, Ber. 70 B, 425 (1937).

[57] F. J. Linnig and R. E. Florin, unpublished work, NBS.

(Paper 65A1-88) 


\section{Publications of the National Bureau of Standards*}

\section{(Including papers in outside journals)}

\section{Selected Abstracts}

Magnetic polarizability of a short right circular conducting cylinder, T. T. Taylor, J. Research NBS 64B, No. 4, 199 (1960).

The magnetic polarizability tensor of a short right circular conducting cylinder is calculated in the principal axes system with a uniform quasi-static but nonpenetrating applied field. One of the two distinct tensor components is derived from results already obtained [1] in connection with the electrical polarizability of short conducting cylinders. The other is calculated to an accuracy of four to five significant figures for cvlinders with radius to half-length ratio of 1/4, 1/2, 1,2 , and 4 . These results, when combined with the corresponding results for the electric polarizability, are applicable to the problem of calculating scattering from cylinders and to the design of artificial dispersive media.

Accuracy of Monte Carlo methods in computing finite Markov chains, N. W. Bazley and P. J. Davis, J. Research NBS 64B, No. 4, 211 (1960).

Experiments are made with the Markov chain presented by the children's game of Chutes and Ladders. Statistics, such as the average length of play, are computed on the IBM 704 from $2^{14}$ simulated plays of the game. These Monte Carlo results are then compared with the "exact solution" obtained by powering the matrix of transition probabilities. Convergence is shown to obey the familiar " $\mathrm{N}^{-\frac{1}{2}}$ " law.

Error bounds in the Rayleigh-Ritz approximation of eigenvectors, H. F. Weinberger, J. Research NBS 64B, No. 4, $217(1960)$

The difference between any eigenvector $u_{p}$ of a linear operator $\mathrm{A}$ and its Rayleigh-Ritz approximation $w_{p}$ is bounded in terms of the differences between the eigenvalues $\lambda_{i}$ of $\mathrm{A}$ and their Rayleigh-Ritz upper bounds $\kappa_{i}$. The bound for the difference between $u_{p}$ and $w_{p}$ approaches zero with $\kappa_{p}$ $-\lambda_{p}$

Numerical solution of the frequency equations for the flexural vibration of cylindrical rods, W. E. Tefft, J. Research NBS 64B, No. 4, 237 (1960).

A numerical solution of Pickett's frequency equations for the flexural vibration of cvlindrical rods, based on the threedimensional differential equations of elasticity, has been obtained on the IBM 704 computer. The results are presented in the form of tables of correction factors to be applied to the thin rod approximation for the fundamental flexural resonance frequency and the first two overtones. These results provide an accurate means of calculating Young's modulus from the density, dimensions, and resonance frequencies of cylindrical rods having diameter-to-length ratios as high as 0.6 .

Incoherent Scattering by Free Electrons as a Technique for Studying the Ionosphere and Exosphere: Some Observations and Theoretical Considerations, K. L. Bowles, J. Research NBS 65D, No. 1, 1 (1961).

Incoherent scattering by the free electrons of the ionosphere has been suggested as a technique for measuring the electron density profile both below and above the $F$ region maximum. This paper reports observations which confirm the existence of the incoherent scatter and show that its intensity is essentially the predicted value. The observed Doppler broadening is considerably smaller than predicted. In the second part of the paper, an explanation for the reduced Doppler broadening is offered. The scatter is explained as arising from statistical fluctuations of electron density, the distribution of which is controlled by the positive ions.
On the Theory of Diffraction by a Composite Cylinder, R. D Kodis, J. Research NBS 65D, No. 1, 19 (1961).

Formulas are developed for the diffracted field around a perfectly conducting cylinder with a dielectric sleeve of arbitrary thickness. These formulas represent the field due to a unit electric line source parallel to the cylinder (either inside or outside the dielectric sleeve) as a spectrum of radial eigenfunctions. It is shown that in each case the field in the region containing the source can be expressed as the sum of two terms, the first of which is a spectral representation of the field when the outer as well as the inner surface of the dielectric is a perfectly conducting boundary. The second term of the sum, which alone involves the properties of the dielectric, is an integral that converges rapidly at high frequencies. Using these general results, perturbation calculations are carried out for three limiting examples of plane wave scattering: (1) thin dielectric; (2) low refractive index; (3) very small surface curvature. In the latter approximation the correspondence with optical results is shown.

\section{A New Approach to the Mode Theory of VLF Propagation,} J. R. Wait, J. Research NBS 65D, No. 137 (1961).

An attempt is made in this paper to present a concise derivation of the mode theory of VLF propagation. Taking note of the fact that the important modes for long distance propagation are near grazing, suitable approximate forms of the wave-functions are introduced at the outset, rather than at the end, of the analysis. It is thus possible to account for the influence of earth curvature in a relatively concise manner. The influence of the earth's magnetic field is also discussed. Finally, numerical results for the attenuation and the phase velocity of the dominant mode are presented.

Bounds on the dissipation of energy in steady flow of a viscous incompressible fluid around a body rotating within a finite region, E. A. Kearsley, Arch. Rational Mech. and Analysis 5, No. 4, 347-354 (1960).

In this paper, bounding techniques are applied to the problem of steady flows of incompressible Navier-Stokes fluids. In particular the torque on a surface of revolution rotating about its axis of symmetry in a finite fixed container of fluid is considered. A Reynolds' number is defined in terms of which the error of the Stokes slow-flow value of torque is rigorously bounded.

Sealed-off $\mathbf{H g}^{198}$ atomic-beam light, source, R. L. Barger and K. G. Kessler, J. Opt. Soc. Am. 50, No. 7, 651-656 (July 1960).

A sealed-off atomic beam light source which utilizes the single isotope $\mathrm{Hg}^{198}$ is described. The emitted $2537 \mathrm{~A}$ line was investigated interferometrically with Fabry-Perot interferometers. Interferograms are shown for retardations of $0.4,1.53$ and 2.04 meter with order numbers 1.6, 6.0 and 8.1 million, respectively. For each retardation, the theoretical contour of the observed fringes is shown. Theoretically predicted fringe contours are shown for retardations up to 6 meter, the approximate limit of interference. It is concluded from the interferograms that the $\mathrm{Hg}^{198} 2537 \mathrm{~A}$ line has a half-width of $0.0016 \mathrm{~cm}^{-1}$, as compared to $0.012 \mathrm{~cm}^{-1}$ for the $\mathrm{Kr}^{86} 6056$ A line proposed as the new primary standard of length. Due to this small half-width and the extremely low level of perturbation in the atomic beam, this $\mathrm{Hg}^{198}$ line would be suitable for the primary standard of length.

Low-energy photoproduction of neutral mesons from complex nuclei, R. A. Schrack, S. Penner, and J. E. Leiss, II Nuovo Cimento 16, Serie X, 759-761 (March 1960).

Angular distribution measurements have been made of neutral pion photoproduction from $\mathrm{C}, \mathrm{Al}, \mathrm{Cu}, \mathrm{Cd}$, and $\mathrm{Pb}$ using 170 
Mev bremsstrahlung. The pion decay photons were detected in coincidence. The observed angular distributions show a diffraction pattern whose first fall-off agrees well with Born approximation prediciions based on coherent production from nuclear matter distributions having the same half density radius as that of the charge distributions determined by electron scattering. The second maxima of the diffraction patterns differ from the Born approximation predictions, the lower A nuclei having the second maxima increasingly enhanced. Analysis of the data has not been completed but the ratio of the observed counting rate to a Born approximation prediction goes as $\mathrm{A}^{1.85}$ where a simple coherent production model yields a ratio of $\mathrm{A}^{2}$.

Electric current and fluid spin created by the passage of magnetosonic wave, R. P. Kanawal and C. Truesdell, Arch. Rational Mech. and Analysis, 5, No. 5, 432-439 (1960).

A general exact theory of weak discontinuities in ionized gases is constructed. Emphasis is put upon the connection between the electric current and fluid vorticity carried by the wave.

Optical constants of aluminum, H. Mendlowitz, Proc. Phys.

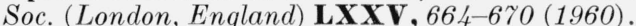

The optical constants of aluminum from the near ultra-violet $(2500 \AA)$ to the near infra-red $(5 \mu)$ can be described, at least qualitatively, in terms of the Drude formula by the two parameters $N$ and $\tau$, the density of free electrons and the relaxation time, respectively. The value for $N$ is taken to correspond to $2 \cdot 4$ free electrons per atom and the value for $\tau$ is taken to be $1 \cdot 2 \times 10^{-15}$ second.

Optical transmissivity and characteristic energy losses, H. Mendlowitz, J. Opt. Soc. Am. 50, No. 7, 739-740 (July $1960)$.

The energy for the onset of transmission of electromagnetic radiation through thin metallic films and the characteristic energy losses of electrons are discussed in terms of the frequency dependence of the complex dielectric constant. The threshold energy for electromagnetic transmission corresponds to that energy for which the real part of epsilon changes from negative to positive, while the prominent characteristic electron absorption peaks should be associated with energies for which the real part of epsilon has just become positive and is still small and the imaginary part of epsilon is very small. Therefore, the threshold energy for optical transmission should be lower than the energy at which the electron absorption occurs.

Optical methods for negative ion studies, S. J. Smith and L. M. Branscomb, Rev. Sci. Instr. 31, No. 7, 733-747 (July 1960).

A high-vacuum crossed-beam apparatus for the study of photodetachment of electrons from negative ions is described, with emphasis on (1) the optical system which transmits a filtered high-intensity photon beam, (2) a high transmission mass selector and associated ion optics, and (3) the sensitive a.c. preamplifier, amplifier, and phase sensitive detector used for measuring the photodetached electron current. The methods used for calibrating and operating the apparatus are discussed.

Nickel oxide thin film resistors for low pressure shock wave detection, K. E. McCullon, Rev. Sci. Instr. 31, No. 7, 780 781 (July 1960).

Shock wave detectors of the resistance-thermometer type, employing thin films of nickel oxide, have been developed and used for shock speed determination in a low-pressure shock tube. In constant-current operation, sensitivity (referred to wall temperature changes) exceeding $30 \mathrm{mv}$ per deg. $\mathrm{C}$ is realized, with a response time less than $1 \mu \mathrm{sec}$.

Casimir coefficients and minimum entropy production, R. E. Nettleton, J. Chem. Phys. 33, No. 1, 237-241 (July $1960)$.

The rate equations obeyed by scalar relaxation parameters in a fluid are extended to include inertial terms in the form of second-order time derivatives, and the relations containing these terms are then written formally as first-order equation: by treating first-order time derivatives as additional param eters. The resulting equations are interpreted thermodynamically as phenomenological relations containing anti symmetric Casimir coefficients, and this interpretation leads with application of the Onsager-Casimir reciprocity theorem to an additional set of phenomenological equations which reduces to those used in an earlier relaxation theory when inertial effects are neglected. In this way, earlier formulat for the bulk viscosity and high-frequency bulk modulus are recovered unchanged. It is also shown why Prigogine's minimum entropy production theorem should no longer hold when one considers inertial effects.

Variations of surface tension calculated with improved ap proximation for activity coefficient, L. C. Shepley and A. B Bestul, J. Am. Ceram. Soc. 43, No. 7, 386-387 (July 1960) The relation in electrolytic solution between the variation 0 surface tension, $\gamma$, with body concentration, $c$, and the variation, $\sigma$, of $\gamma$ with surface concentration, $c_{s}$, has previously been examined using the crude approximation that the activity coefficient $g$ of the solute is a linear function of concentration. This note examines the above relation using $d$ more nearly adequate approximation for $g$, which is suggested directly by the Debye-Hückel Limiting Law for the activity coefficient of electrolytes in solution. The results confirm the previous conclusions that the derivative $\alpha^{2} \gamma / d \sigma d c$ must have negative values for certain conditions of large $\sigma$ and small $c$.

The extent of $\mathbf{H}_{\text {II }}$ Regions, S. R. Pottasch, Astrophys. $J$ 132, No. 1, 269-271 (July 1960).

For an atmosphere whose electron density is less than $10^{5}$ $\mathrm{cm}^{-3}$, and which is thin in all Balmer and higher radiation fields (conditions which may be fulfilled in planetary and diffuse nebulae) it is shown that the extent of the ionized region can be found using the formula given by Strömgren (1939) multiplied by a correction factor which depends only on the temperature of the exciting star. This factor is given in this note.

Some aspects of fluorine flame spectroscopy, D. E. Mann Proc. Propellant Thermodynamics and Handling Conf. Specia Rept. 12, (Ohio State University, Columbus, Ohio, J une 1960). Recent studies in the spectroscopy of flames supported by fluorine or chlorine trifluoride are reviewed, and a description of the experimental procedures currently in use is given Certain problems and recent results of special interest are discussed. These include considerations bearing on the occurrence of $\mathrm{CF}$ in hydrocarbon-fluorine flames and the presence of a singlet system of $\mathrm{NH}$ in the emission spectrum of the ammonia-fluorine flame. The recently completed investigation of the hydrogen-fluorine flame with high dispersion is described. Some of the spectroscopic constants of $\mathrm{HF}$ established by these measurements are presented for the first time.

Current thermodynamic research on light-element compounds at the National Bureau of Standards, T. E. Douglas. Proc. Propellant Thermodynamics and Handling Conf. Special Rept. 12, (Ohio State University, Columbus, Ohio, June 1960). As part of a broader program on properties of materials at high temperatures now required in many technical and scientific fields, an experimental and theoretical investigation of the thermodynamic properties of light-element compounds is now underway at the National Bureau of Standards. This research program presently comprises the elements lithium, beryllium, magnesium, and aluminum, free and in chemical combination with one or more of the elements hydrogen, nitrogen, oxygen, fluorine, and chlorine. It has as its objective the securing of the basic data which are necessary to determine, with an accuracy of one percent where possible, the energies and equilibrium proportions of these substances in the solid, liquid and gaseous states in the temperature range from $0^{\circ}$ to $6000^{\circ} \mathrm{K}$ and the pressure range from 0 to 100 atmospheres. Nine research groups are involved. Their individual activities and plans for future work are outlined in some detail. These groups may be described by the titles 
1) fluorine calorimetry, (2) thermochemistry, (3) lowemperature calorimetry, (4) high-temperature calorimetry and halide equilibria, (5) statistical thermodynamies and computer codes, (6) high-temperature high-pressure transient phenomena, (7) molecular structure from spectra, (8) thermodynamics of refractory substances, and (9) chemical preparaion and properties of hydrides.

Structure of Sulfurous Esters, H. Finegold, Proc. Chem. Soc. (London) 283-284 (A ugust 1960).

Anomalies in the proton magnetic resonance spectra of sulfurous acid esters are discussed. These features suggest a undamental skewness in the nature of the bonding orbitals emanating from the central chalcogen atom. The implications of such a theory are mentioned.

Temperature dependence of Young's modulus of vitreous germania and silica, S. Spinner and G. W. Cleek, J. Appl. Phys. 31, No. 8, 1407-1410 (1960).

The temperature dependence of Young's Modulus of vitreous $\mathrm{GeO}_{2}$ has been determined by a dynamic resonance method from $-195{ }^{\circ} \mathrm{C}$ to $540{ }^{\circ} \mathrm{C}$. The modulus increases with increasing temperature from about $-120{ }^{\circ} \mathrm{C}$ to $400{ }^{\circ} \mathrm{C}$. Below and above this range the modulus decreases with increasing temperature. Young's modulus for vitreous $\mathrm{SiO}_{2}$ also increases from about $-190^{\circ} \mathrm{C}$ to $1,175{ }^{\circ} \mathrm{C}$ and decreases with increasing temperature outside this range.

In view of the similarity in structures and bond energies of these two materials, the similarity in the elastic modulustemperature relations is believed to be significant; especially when contrasted with the lack of agreement in another commonly measured anharmonic property, thermal expansion.

Photolysis of ammonia in a solid matrix at low temperatures O. Schnepp and K. Dressler, J. Chem. Phys. 32, No. 6, 16821686 (June 1960).

Solid deposits of argon containing 0.3 mole percent ammonia were irradiated at $4.2^{\circ} \mathrm{K}$ with light of wavelengths shorter than $2000 \mathrm{~A}$. The emission of a hydrogen discharge with a LIF window and of a thin-walled quartz mercury arc were used. The production of the unstable species $\mathrm{NH}$ and $\mathrm{NH}_{2}$ was observed by means of electronic absorption spectroscopy. Experiments using filters led to the conclusion that $\mathrm{NH}$ is produced by irradiation with light of wavelengths shorter than $1550 \mathrm{~A}$. $\mathrm{NH}_{2}$ is produced by radiation above $1700 \mathrm{~A}$ and below $1550 \mathrm{~A}$ with comparable quantum efficiency. Warm-up experiments show that $\mathrm{NH}_{2}$ disappears close to $20^{\circ} \mathrm{K}$ whereas $\mathrm{NH}$ is stable up to at least $30^{\circ} \mathrm{K}$. Photolysis at $20^{\circ} \mathrm{K}$ is approximately five times less efficient than at $4.2^{\circ} \mathrm{K}$. On certain assumptions a molar absorption coefficient of 40,800 is estimated for both $\mathrm{NH}$ and $\mathrm{NH}_{2}$ and the $f$-values of the observed transitions of these molecules are estimated to be of the order of $10^{-3}$.

Isotope effect in the hydrogen atom-formaldehyde reaction, J. R. McNesby, M. D. Scheer, and R. Klein, J. Chem. Phys. 32, No. 6, 1814-1817 (June 1960).

The isotope effect in the hydrogen abstraction from formaldehyde by hydrogen atoms has been measured. The activation energy difference derived from relative rate measurements of the pair of reactions

$$
\begin{aligned}
& \mathrm{H}+\mathrm{H}_{2} \mathrm{CO} \rightarrow \mathrm{H}_{2}+\mathrm{HCO}(2) \\
& \mathrm{H}+\mathrm{D}_{2} \mathrm{CO} \rightarrow \mathrm{HD}+\mathrm{DCO}(5)
\end{aligned}
$$

is $\mathrm{E}_{5}-\mathrm{E}_{2}=1.0 \mathrm{kcal}$. The value for the corresponding pair,

$$
\begin{aligned}
& \mathrm{D}+\mathrm{H}_{2} \mathrm{CO} \rightarrow \mathrm{HD}+\mathrm{HCO}(6) \\
& \mathrm{D}+\mathrm{D}_{2} \mathrm{CO} \rightarrow \mathrm{D}_{2}+\mathrm{DCO}(7)
\end{aligned}
$$

$\mathrm{E}_{7}-\mathrm{E}_{6}=0.9 \mathrm{kcal}$., is calculated from (5), (2) and the variation of the hydrogen-deuterium equilibrium constant with temperature. Application of the Biegeleisen theory of the isotope effect to these reactions suggests a loosely bound activated complex.
Absorption spectra of solid xenon, krypton, and argon in the vacuu m ultraviolet, O. Schnepp and K. Dressler, J. Chem. Phys. 33, No. 1, 49-55 (July 1960).

The absorption spectra of solid xenon, krypton and argon at $4 .{ }^{\circ} \mathrm{K}$ have been investigated between $3500 \mathrm{~A}$ and $1200 \mathrm{~A}$. In the region between $1510 \mathrm{~A}$ and $1200 \mathrm{~A}$ solid xenon has four absorption bands, three of which lie within less than $800 \mathrm{~cm}^{-1}$ of atomic transitions, all being shifted to lower energy in the solid. Solid krypton has two bands between $1250 \mathrm{~A}$ and $1200 \mathrm{~A}$ which lie within $900 \mathrm{~cm}^{-1}$ of atomic transitions but are shifted to higher energy in the solid. No absorption was found in solid argon at wavelengths longer than $1200 \mathrm{~A}$. The experimental results are interpreted and discussed on the basis of valence type interactomic interactions in the excited states. It is predicted that the fluorescence spectra of these solids would be displaced to lower energy by about 1 e.v.

Absorption spectra of solid methane, ammonia, and ice in the vacuum ultraviolet, $K$. Dressler and O. Schnepp, J. Chem. Phys. 33, No. 1, 270274 (July 1960).

The spectra of solid methane, ammonia and ice were investigated in the vacuum ultraviolet region. The spectrum of solid methane is very similar to that of the gas but the spectra of solid ammonia and ice are shifted to shorter wavelengths relative to the corresponding vapor spectra by $7500 \mathrm{~cm}^{-1}$ and $7000 \mathrm{~cm}^{-1}$ respectively. The effect of hydrogen bonding in the ground state of solid ammonia and ice is discussed and it is suggested that the lowest excited states are raised in energy relative to the gas phase levels due to repulsive intermolecular interactions.

Free radicals in gamma-irradiated polystyrenes, R. E. Florin, L. A. Wall, and D. W. Brown, Trans. Faraday Soc. 56, No. 453, 1304-1310 (Sept. 1960).

Electron spin resonance spectra were observed for $\gamma$-irradiated polystyrene and for a series of substituted polystyrenes $\alpha-d_{1}, \quad \beta-d_{1}, \quad p-d_{1}, \quad \beta, \quad \beta-d_{2}, \quad \alpha, \quad \beta, \quad \beta-d_{3}, \quad m-\mathrm{CH}_{3}, \quad \alpha-\mathrm{CH}_{3}$, $2,5-\mathrm{Cl}_{2}$, and $\alpha, \beta, \beta-\mathrm{F}_{3}$. The spectra of all the $d$-substituted styrenes irradiated at $77^{\circ} \mathrm{K}$ were alike, consisting of three peaks with a separation of 37 gauss between center and end derivative peaks. The result is consistent with a radical structure formed by removal of a hydrogen but the major hyperfine interaction is with ortho ring hydrogens. The same structure seems to apply for poly (m-methylstyrene) and $\operatorname{poly}(\alpha, \beta, \beta$-trifluorostyrene), but in poly (2,5-dichlorostyrene) all hyperfine interactions are much lower. The radical from poly $(\alpha$-methylstyrene $)$ is formed by main chain scission and shows evidence for relatively free rotation of the $\alpha$-methyl group.

Halobenzenes as sensitizers for the radiation-induced polymerization of styrene, D. W. Brown and L. A. Wall, J. Polymer Sci. 44, 325-340 (June 1960).

Halogenated benzenes, carbon tetrachloride, and other compounds have been used to sensitize the gamma-ray-induced polymerization of styrene. Benzene and fluorobenzenes are about equally effective while the data from solutions of mono- and di-halogenated benzenes indicate that the order of increasing sensitivity to radiation is: Fluorobenzenes $<$ chlorobenzenes < bromobenzenes < iodobenzenes. One halogen is less effective than two, and the ortho isomers are more effective than the meta and para. The relations between the $G$-values for radical production in the solutions and the electron fractions of solvent are linear only for benzene and the monohalogenated aromatics. With very highly halogenated benzenes and carbon tetrachloride the departure from linearity is very marked. Significant sensitization is observed at very low electron fractions with the highly halogenated materials, except with hexafluorobenzene, which appears to be very stable to radiation. The generalizations concerning the effects of the kind and number of substituents in monoand di-halogenated benzenes are not valid for more highly halogenated benzenes. Much of the curvature observed at high concentrations of carbon tetrachloride is apparently due to changes in the rate constants as the monomer concentration is changed. At low concentrations this is not the case with carbon tetrachloride and presumably also with the other highly halogenated materials. Various reaction mech- 
anisms are considered and one is advanced that reproduces many of the important features of the observed data.

Pyrolysis of polyolefins, L. A. Wall and S. Straus, J. Polymer Sci. 44, 313-323 (J une 1960).

The differences in thermal decomposition behavior previously observed between linear and branched polyethylenes have been explored experimentally by investigating and comparing the rates of volatilization of a series of twenty polymers. Similar differences were observed between linear and branched polypropylenes. The linear material in both cases exhibited behavior characteristic of a random decomposition, while the branched material did not. Other materials studied included high-pressure polyethylene; low pressure copolymers of ethylene with propene, butene, and penten-1; and polymers and copolymers prepared from diazoalkanes. Systematic deviations from random theory were found to occur with branching. The greater the branching, provided that the branches were longer than one carbon atom, the greater was the rate of decomposition and the more it was at variance with random therory. No quantitative theory accounting for these results is yet available. However, it seems that polymer branching enhances intramolecular transfer at the expense of intermolecular transfer. This is evidenced by the observed type of their rate-versus-conversion curves, which are similar to those found for polymers that mainly decompose into monomer.

\section{Other NBS Publications}

\section{Journal of Research, Section 64B, No. 4, October-December}

1960. 75 cents.

Magnetic polarizability of a short right circular conducting cylinder, T. T. Taylor. (See above abstract.)

Accuracy of Monte Carlo methods in computing finite Markov chains, N. W. Bazley and P. J. Davis. (See above abstract.) Error bounds in the Rayleigh-Ritz approximation of eigenvectors, H. F. Weinberger. (See above abstract.)

Sequence transformations based on Tchebycheff approximations, J. R. Rice.

Numerical solution of the frequency equations for the flexural vibrations of cylindrical rods, W. E. Tefft. (See above abstract.)

Journal of Research, Section 65D, No. 1, January-February 1961. 70 cents.

Incoherent scattering by free electrons as a technique for studying the ionosphere and exosphere: some observations and theoretical considerations, K. L. Bowles. (See above abstract.)

Radio wave absorption of several gases in the 100 to 117 $\mathrm{kMc} / \mathrm{s}$ frequency range, C. O. Britt, C. W. Tolbert, and A. W. Straiton.

On the theory of diffraction by a composite cylinder, R. D. Kodis. (See above abstract.)

An atlas of oblique-incidence ionograms (a digest), V. Agy, K. Davies, and R. Salaman.

A new approach to the mode theory of VLF propagation, J. R. Wait. (See above abstract.)

East-west effect on VLF mode transmission across the earth's magnetic field, D. Dobrott and A. Ishimaru.

Magneto-ionic propagation phenomena in low- and very-lowradiofrequency waves reflected by the ionosphere, J. R. Johler.

Correlation of monthly median transmission loss and refractive index profile characteristics, B. R. Bean and B. A. Cahoon.

Characteristics of waveguides for long-distance transmission, A. E. Karbowiak and L. Solymar.

Useful radiation from an underground antenna, H. A. Wheeler. Observation of F-layer and sporadic-E scatter at VHF in the Far East, K. Miya, T. Sasaki, and M. Ishikawa.

A high-resolution rapid-scan antenna, H. V. Cottony and A. C. Wilson.

Alphabetical index to tables of chemical kinetics. Homogeneous reactions, Supplement 2 to NBS Circ. 510 (1960) 35 cents.

The metric system of measurement, NBS Misc. Publ. 232 (1960), 50 cents.
Standard X-ray diffraction powder patterns, H. E. Swanson, M. I. Cook, E. H. Evans, and J. H. deGroot. NBS Circ. 539, Vol. 10 (1960) 40 cents.

Stabilization of free radicals at low temperatures, edited by A. M. Bass and H. P. Broida. NBS Mono. 12 (1960) $\$ 1.50$ Protection against radiations from sealed gamma sources. NB Handb. 73 (1960) (Supersedes H54) 30 cents.

Standardization activities in the United States. A descriptive directory, S. F. Booth. NBS Misc. Publ. 230 (1960) (Supersedes M169) \$1.75.

Hydraulic Research in the United States, 1960, H. K. Middleton. NBS Misc. Publ. 231 (1960) \$1.00.

Investigation of bearing creep of two forged aluminum allovs, L. Mordfin, N. Halsey, P. J. Granum. NBS TN55 (PB161556) (1960) \$1.00.

A survey and bibliography of recent research in the propagation of VLF radio waves, J. R. Wait. NBS TN58 (PB161559) (1960) 75 cents.

Amplitude and phase of the low- and very low-radiofrequency ground wave, J. R. Johler, L. C. Walters, and C. M. Lilley. NBS TN60 (PB161561) (1960) 75 cents.

Rapid determination of the order of chemical reactions from time-ratio tables, J. H. Flynn. NBS TN62 (PB161563) (1960) 75 cents.

Single scattered neutrons from an isotropic point source, E. R. Mosburg, Jr., and W. M. Murphey. NBS TN63 (PB161564) (1960) 50 cents.

Radio Refractometry, J. W. Herbstreit. NBS TN66 (PB161567) (1960) 50 cents. Low- and very low-radiofrequency model ionosphere reflection coefficients, J. R. Johler, L. C. Walters, J. D. Harper, Jr., NBS TN69 (PB161570) (1960) \$2.00.

Clay mineral content of two domestic kaolins, W. C. Ormsby and J. M. Shartsis, J. Am. Ceram. Soc. 43, No. 6, 335 (June 1960).

Some Canons of sound experimentation, C. Eisenhart, Bull. Inst. Intern. Stat. XXXVII, No. 3, 339-350 (1960).

Thermodynamic properties of helium at low temperatures and high pressures, D. B. Mann and R. B. Stewart, Trans. Am. Soc. Mech. Engrs. J. Heat Transfer 81, No. 4, 324 326 (Nov. 1959).

Interpolation of platinum resistance thermometers, $20^{\circ}$ to to $273.15^{\circ} \mathrm{K}, \mathrm{R}$. J. Corruccini, Rev. Sci. Instr. 31, No. 6, 637-640 (June 1960).

Radiation from a slot on a large corrugated cylinder, J. R. Wait and A. M. Conda, Electromagnetic Wave Propagation, Proc. Intern. Conf. Propagation of Radio Waves, Liege, Belgium, 1958, p. 103 (Academic Press, Inc., New York, N.Y., 1960).

Low-temperature transport properties of commercial metals and alloys. IV. Reactor grade $\mathrm{Be}, \mathrm{Mo}$, and $\mathrm{W}, \mathrm{R}$. L Powell, J. L. Harding, and E. F. Gibson, J. Appl. Phys. 31, No. 7, 1221-1224 (July 1960).

A rating scale method for evaluating research positions, $\mathrm{H}$. A. McKean, J. Mandel, and M. N. Steel, Personnel Admin. 23, No. 4, 29-36 (July-Aug. 1960).

Post office mechanization, B. M. Levin, M. C. Stark, and P. C. Tosini, Proc. Information Processing and Retrieval, Am. Inst. Elec. Engrs. General Meeting, June 19-24, 1960, Atlantic City, N.J., Am. Inst. Elec. Engrs. Paper CP60-937 (1960).

Sensitive thermal conductivity gas analyzer, J. R. Purcell and R. N. Keeler, Rev. Sci. Instr. 31, No. 3, 304-306 (March 1960)

A study of two water resistance testers for shoe upper leather, R. L. Hebert, J. Am. Leather Chemists Assoc. LV, No. 7, 388-405 (July 1960).

Atomic clocks for space experiments, P. L. Bender, Astronautics p. 69-71 (July 1960).

Photochemical rates in the equatorial $\mathrm{F}_{2}$ region from the 1958 eclipse, T. E. Van Zandt, R. B. Norton, and G. H. Stonehocker, J. Geophys. Research 65, No. 7, 2003-2009 (July, 1960).

Influence of source distances on the impedance characteristics of ELF radio waves, J. R. Wait, Proc. IRE 48, No. 7, 1338 (July 1960).

Electroless plated contacts to silicon carbide, R. L. Raybold. Rev. Sci. Instr. 31, No. 7, 781-782 (July 1960). 
Statistical models for component aging experiments, J. R. Rosenblatt. Intern. Conv. Record. Inst. Radio Engrs. 8, Pt. 6, 115-124 (1960).

The nature of the inorganic phase in calcified tissues, A. S. Posner. Calcification in Biological Systems, p. 373-394 (American Assoc. Advancment of Sci., Washington, D.C., 1960).

Effect of water-reducing admixtures and set-retarding admixtures on properties of concrete, Introduction and Summary, B. E. Foster. Am. Soc. Testing Materials Spec. Tech. Publ. 266, Introduction 1 \& 2 and Summary 240246 (June 1960).

Council adopts F.D.I. specification for alloy for dental amalgam, Council on Dental Research, J. Am. Dental Assoc. 60, No. 6, 773 (June 1960).

The foundations of mechanics and thermodynamics, E. A. Kearsley and M. S. Green, Phys. Today 13, No. 7, 22-25 (July 1960).

On the theory of the slow-tail portion of atmospheric waveforms, J. R. Wait, J. Geophys. Research 65, No. 7, 19391946 (July 1960).

Statistical aspects of the cement testing program, W. J. Youden. Am. Soc. Testing Materials Proc. 59, 11201128 (1959).

Transfer of liquid hydrogen through uninsulated lines, R. J. Richards, W. G. Steward, and R. B. Jacobs, Proc. 1959 Cryogenic Engr. Conf., Vol. 5, Advances in Cryogenic Engineering, p. 103 (Plenum Press, New York, N.Y., 1960).

Low and medium frequency radio propagation, K. A. Norton, Electromagnetic Wave Propagation, Proc. Intern. Conf. Propagation of Radio waves, Liege, Belgium, 1958, p. 375 (Academic Press Inc., New York, N.Y., 1960).
Cavity resonator dielectric measurements of rod samples, H. E. Bussey, Conf. Electrical Insulation (Annual Report of 1959), Natl. Acad. Sci., Natl. Research Council Publ. 756, 15-20 (1960).

Analysis of fractionally replicated $2^{m} 3^{n}$ designs, R. C. Bose and W. S. Connor, Bull. Inst. Intern Stat XXXVI, No. 3, 141-160 (1960).

A barium fluoride film hygrometer element, F. E. Jones and A. Wexler, J. Geophys. Research 65, No. 7, 2087-2095 (July 1960).

Radiation patterns of finite-size corner-reflector antennas, A. C. Wilson, H. V. Cottony, IRE Trans. Ant. Prop. AP-8, No. 2, 144-157 (Mar. 1960).

VLF attenuation for east-west and west-east daytime propagation using atmospherics, W. L. Taylor, J. Geophys. Research 65, No. 7, 1933-1938 (July 1960).

Mechanized conversion of colorimetric data to munsell renotations, W. Rheinboldt and J. P. Menard, J. Opt. Soc. Am. 50, No. 8, 802-807 (Aug. 1960).

The use of geostationary satellites for the study of ionospheric electron content and ionospheric radio-wave propagation, O. K. Garriott and C. G. Little, J. Geophys. Research 65, No. 7, 2025-2027 (July 1960).

Publications for which a price is indicated (except for NBS Technical Notes) are available only from the Superintendent of Documents, U.S. Government Printing Office, Washington 25, D.C. (foreign postage, one-fourth additional). Technical Notes are available only from the Office of Technical Services, U.S. Department of Commerce, Washington 25, D.C. (order by PB number). Reprints from outside journals and the NBS Journal of Research may often be obtained directly from the authors. 